\title{
Processus de transfert atmosphérique de polluants dans les agrosystèmes
}

\author{
Atmospheric transport processus of pollutants in agrosystems
}

\author{
par P. Cellier
}

Institut National de la Recherche Agronomique

Unité de Recherche en Bioclimatologie, Thiverval-Grignon

Different pollutants, either gaseous or particulate, are emitted in the atmosphere from agricultural systems. The sources may be farm housings, field application and volatilisation from the soil or the plant. These emissions are a loss of nutrients for the agricultural systems but also a source of pollution for the atmosphere and for the neighbouring ecosystems or surface water after deposition.

The different processes involved in pollutant emission, atmospheric transport and deposition are reviewed. Emission occurs in different conditions : direct injection of particulates or droplets from animal housing or during field application of fertilisers or pesticides, and gas production resulting from either physico-chemical equilibria or microbial metabolism. For both gaseous or particulate compounds, deposition is strongly dependent on the surface characteristics (leaf area index, wetness, roughness, metabolism). The deposition is usually determined by estimating a deposition velocity which accounts for the effects of turbulence, canopy processes and the pollutant characteristics. Atmospheric diffusion and transport processes make a link between the source (emission) and the sink (deposition) of pollutants. They are both dependent on meteorological conditions and surface characteristics. Then estimating atmospheric deposition at regional scale requires a coupling between emission, transport and deposition models.

\section{INTRODUCTION}

Depuis le milieu du vingtième siècle, l'agriculture fait une utilisation de plus en plus intensive d'intrants, tels que les engrais minéraux (urée, ammonitrate, solutions azotées) ou organiques (lisiers, fumiers, effluents urbains ou agro-industriels), et les produits phytosanitaires (fongicides, herbicides, insecticides, qu'on mentionnera dans la suite sous le terme général " pesticide "). Cependant une partie non négligeable de ces produits peut être perdue vers l'atmosphère : les pertes d'azote par volatilisation d'ammoniac peuvent aller de 5 à $50 \%$ des apports après utilisation d'engrais minéraux, et peuvent dépasser $50 \%$ après épandage de lisier [1]. Des chiffres similaires peuvent être trouvés pour les produits phytosanitaires, avec une grande variabilité résultant de la variété de formulations et de volatilité de ces produits $[2,3$, 4]. Pour Paterson et al. [5], l'atmosphère est d'ailleurs l'une des voies principales de dissémination des pesticides vers l'homme et les écosystèmes naturels.

D'un point de vue strictement agronomique, ces pertes vers I'atmosphère représentent un manque à gagner. Lorsqu'on s'intéresse au problème de la pollution des eaux, on pourrait penser que ces pertes sont bénéfiques car elles minimisent les risques de migration de ces composés vers les eaux. Cependant, une fois dans l'atmosphère, ces composés sont transportés rapidement et à grande distance puis, s'ils ne sont pas dégradés, sont déposés sur d'autres écosystèmes. Ces dépôts atmosphériques sont, pour beaucoup d'écosystèmes naturels, la principale source d'éléments minéraux [6]. Après dépôt, ces composés sont réintégrés dans le cycle de l'eau, et le problème de leur impact et de leur devenir se pose alors en d'autres lieux que celui où ils ont été appliqués. Sur des sols très filtrants, la migration vers les eaux peut être plus rapide que sur les sols agricoles où a été fait l'épandage. On sait de plus que le dépôt de composés acidifiant le sol tels que l'azote ammoniacal peut favoriser la remise en solution et le lessivage de certains cations comme l'aluminium ou le magnésium.

Pour un composé gazeux, le dépôt en un point est d'autant plus important que la concentration dans l'air est élevée. Celle-ci est déterminée par l'intensité des sources (activités industrielles, domestiques ou agricoles, gestion des déchets, transports, milieu naturel), leur proximité et l'efficacité de la dispersion atmosphérique entre la source et le puits. Pour quantifier les dépôts en un point, il faut donc connaître également les processus d'émission dans le voisinage et de 
transport par l'atmosphère jusqu'à ce point. C'est ainsi que les modèles qui calculent les dépôts à l'échelle d'une région ou d'un pays calculent sur la même grille les émissions et les dépôts, les relations entre les deux étant calculées à l'aide d'un modèle de transport, éventuellement couplé à un modèle de transformation chimique dans l'atmosphère $[7,8]$. On doit donc s'intéresser à l'ensemble de la chaîne depuis la source jusqu'au puits, c'est-à-dire : (1) l'émission, ou passage de la source vers l'atmosphère, (2) le transport proprement dit du produit par la turbulence atmosphérique depuis cette source jusqu'à la " cible " et (3) le dépôt sur la " cible". Pour ces trois phases, nous allons présenter les principaux processus de transfert impliqués et quelques possibilités de modélisation, en nous focalisant sur les systèmes agricoles.

\section{II — EMISSION VERS L'ATMOSPHÈRE}

\subsection{Quels composés et quelles sources ?}

Dans les systèmes agricoles, des polluants peuvent être émis en différents endroits (bâtiments, lors de l'application de produits, depuis le sol ou la culture) et sous différentes formes : particules (poussières, gouttelettes, aérosol) ou gaz. Par rapport aux autres sources naturelles ou anthropiques (industries, transports, activités domestiques), la contribution de l'agriculture est dominante pour quelques composés :

- l'ammoniac, produit principalement par les activités d'élevage et la fertilisation : l'agriculture représenterait plus de 95\% des sources en France [9],

- les produits phytosanitaires : aucune quantification précise n'a été faite, mais $80 \%$ de ces produits étant utilisés dans le domaine agricole, on peut supposer que la part des émissions est sensiblement équivalente,

- le méthane, produit essentiellement par la fermentation entérique des ruminants. Sa part est estimé à plus de $60 \%$ pour la France [9],

- le protoxyde d'azote, émis lors des transformations de l'azote dans le sol (nitrification, dénitrification). La proportion émise par les sols naturels ou agricoles serait supérieure à 50\% des émissions en France [9].

Pour des composés tels que les $\mathrm{NO}_{x}$, la contribution globale des sources naturelles et de l'agriculture serait d'environ $25 \%$, résultat de sources de faible intensité, mais portant sur de grandes étendues.

\subsection{Emission depuis un épandeur ou un bâtiment d'élevage}

Lors des épandages, une fraction importante des composés apportés peut se volatiliser avant d'atteindre la surface du sol et de la végétation. Cette fraction dépend de la vitesse du vent, de la volatilité et du fractionnement (taille des gouttelettes) du produit et de variables telles que la température et le $\mathrm{pH}$ qui agissent sur les équilibres entre forme dissoute et forme gazeuse (constante de Henry). Elle est typiquement de 5-10\% pour l'azote ammoniacal lors d'un épandage de lisier [1].

Pour les produits phytosanitaires, une voie importante et rapide de dispersion est l'entraînement par le vent des gouttelettes (de très petite taille) émises depuis les épandeurs [10]. Malgré l'utilisation de rampes de pulvérisation, ces pertes varient de 5 à $30 \%$ [2], voire $50 \%$ lors des épandages aériens. Cet entraînement par le vent constitue une voie de transfert très rapide (quelques secondes) à courte distance, notamment vers les cours d'eau ou fossés bordant les zones agricoles.

Les bâtiments d'élevage et les zones de stockage d'effluents sont des sources de très forte densité d'émission, qui ont un impact majeur sur leur environnement proche. Les composés émis sont essentiellement des gaz azotés $\left(\mathrm{NH}_{3}, \mathrm{~N}_{2} \mathrm{O}, \mathrm{NO}_{\mathrm{x}}\right)$ ou carbonés $\left(\mathrm{CO}_{2}, \mathrm{CH}_{4}\right)$, mais également des gaz soufrés et des poussières qui résultent du métabolisme et de l'activité des animaux [11].

\subsection{Emission depuis la surface du sol ou de la végé- tation vers l'atmosphère}

Deux types de processus peuvent être impliqués dans la production de composés gazeux : les équilibres entre formes dissoutes et gazeuses, et le métabolisme de la biomasse microbienne du sol.

\subsubsection{Equilibres physico-chimiques}

Deux cas typiques sont ceux des émissions d'ammoniac et de pesticides après épandage sur le sol ou la végétation. On peut généralement estimer que le flux de volatilisation ( $F$ en $\left.\mu \mathrm{g} \mathrm{m}^{-2} \mathrm{~s}^{-1}\right)$ est proportionnel à l'écart de concentration $\left(\mu \mathrm{g} \mathrm{m}^{-3}\right)$ entre la surface et l'air à la hauteur $z$.

$$
F=\left[C_{s}-C_{a}\right] / r_{a}(z)
$$

La concentration dans l'atmosphère $\left(C_{a}\right)$ étant en général très faible (quelques $\mu \mathrm{g} \mathrm{m}^{-3}$ voire quelques centaines de $\mathrm{pg} \mathrm{m}^{-3}$ ), le flux est principalement déterminé par la concentration à la surface $\left(C_{s}\right)$ et les conditions de diffusion dans les basses couches atmosphériques ( $r_{a}$ est une résistance aérodynamique, en $\mathrm{s} \mathrm{m}^{-1}$ ). La concentration $C_{s}$ dépend des équilibres entre les diverses formes du composé au niveau du sol ou de la végétation. Un exemple simple, celui de l'ammoniac, est présenté en Figure 1.



1. Schéma d'un modèle de volatilisation d'ammoniac.

Après épandage d'engrais, l'azote ammoniacal est présent dans le sol sous 4 formes : (1) ammoniac gazeux $\left(\mathrm{NH}_{3 g}\right)$, (2) ammoniac dissous $\left(\mathrm{NH}_{3 a q}\right)$, (3) ammonium dissous $\left(\mathrm{NH}_{4}^{+}{ }_{a q}\right)$ et (4) ammonium adsorbé sur les fractions minérales et organiques du sol $\left(\mathrm{NH}_{4}^{+}{ }_{5}\right)$. Les équilibres entre ces différentes formes évoluent rapidement dans le temps sous l'influence de la volatilisation, et de la température et de la teneur en eau des couches de surface du sol.

Pour les pesticides, la volatilisation est l'une des voies principales de fuite hors de la zone cible en particulier pour les traitements de surface $[2,5]$. Elle dépend de la pression de vapeur en pesticide à la surface du sol, de sa solubilité, du degré d'adsorption du pesticide sur les fractions solides du sol, de la température et de la teneur en eau du sol [3,4]. Selon les produits, la solubilité et la volatilité peuvent varier 
d'un facteur $10^{6}$ entre les extrêmes [4], avec quelquefois un ou plusieurs ordres de grandeur pour des molécules très proches.

Dans les deux cas, tout facteur ou variable qui déplace l'un de ces équilibres vers la forme gazeuse favorise la volatilisation. Les facteurs liés au climat sont principalement la température, la pluviométrie et l'évaporation (via la teneur en eau du sol). Les techniques agricoles jouent sur les doses appliquées et la profondeur d'enfouissement du produit.

Les processus se produisant au niveau du sol sont multiples. Il faut tout d'abord prendre en compte les transferts du produit considéré dans le sol, pour estimer ce qui reste à la surface. Ces transferts sont souvent très rapides au moment ou juste après l'application du produit. Ils se produisent sous forme soluté ou gazeuse. Il faut ensuite prendre en compte les interactions physico-chimiques entre le sol et le produit. La proportion d'ammoniac est par exemple très sensible au pH du sol (équilibre $\mathrm{NH}_{4 a q}{ }^{+}-\mathrm{NH}_{3 a q}$ ). En outre, l'azote ammoniacal ou le pesticide se trouvent dans une solution complexe contenant beaucoup de composés (cations, acides gras volatils) qui peuvent interagir avec eux. Enfin, l'adsorption et la fixation sur la fraction solide du sol (éléments minéraux et matière organique) peuvent être très variables selon la texture du sol et sa capacité d'échange cationique. Alors qu'une augmentation de la teneur en eau du sol est connue pour diminuer la volatilisation d'ammoniac par dilution, elle stimule celle de certains pesticides car l'adsorption des molécules de pesticides est plus forte lorsqu'il n'y a pas d'eau au contact des éléments minéraux ou organiques du sol. Ces interactions avec la matière organique et les éléments minéraux du sol sont en outre très dépendantes de la structure moléculaire et de la polarité des pesticides.

Enfin, la diffusion sous forme vapeur ou soluté dans le sol est un processus déterminant pour la volatilisation des pesticides injectés dans le sol. En effet, contrairement aux composés azotés, certains pesticides persistants peuvent rester présents dans le sol sur des durées de plusieurs semaines, ce qui leur laisse le temps de migrer dans le sol vers la surface par diffusion et/ou convection. Des taux de volatilisation de plus de $50 \%$ ont ainsi été mesurés pour des produits de traitement du sol contre les nématodes, apportés à une profondeur de $15 \mathrm{~cm} \mathrm{[3].}$

\subsubsection{Métabolisme microbien}

Les micro-organismes du sol peuvent produire différents composés sous forme gazeuse, comme sous-produits de leur métabolisme. C'est le cas du monoxyde (NO) et du protoxyde $\left(\mathrm{N}_{2} \mathrm{O}\right)$ d'azote, produit par nitrification et dénitrification, du $\mathrm{CO}_{2}$ produit par la respiration des micro-organismes du sol, du méthane $\left(\mathrm{CH}_{4}\right)$ et du sulfure d'hydrogène $\left(\mathrm{H}_{2} \mathrm{~S}\right)$, tous deux produits en conditions très anoxiques. Un exemple bien connu est celui de la production de $\mathrm{N}_{2} \mathrm{O}$, gaz à effet de serre, par dénitrification. Les micro-organismes dénitrifiants - présents dans la plupart des sols - utilisent le nitrate $\left(\mathrm{NO}_{3}{ }^{-}\right)$ comme accepteur d'électron dans leur processus de respiration lorsque la concentration en oxygène dans le sol est limitante, c'est-à-dire le plus souvent en conditions d'excès d'eau. La chaîne de réduction passe de l'ion nitrate, au nitrite $\left(\mathrm{NO}_{2}{ }^{-}\right)$, puis au $\mathrm{N}_{2} \mathrm{O}$ et enfin au $\mathrm{N}_{2}$. Les émissions de gaz par les sols dépendent donc en premier lieu de la capacité de la microflore du sol à produire ou consommer ces gaz. Mais le fonctionnement de cette microflore est sous le contrôle de facteurs abiotiques tels que la température et la teneur en eau du sol. En outre, l'émission réelle à la surface du sol dépend également de la diffusion du composé gazeux depuis son lieu de production jusqu'à l'atmosphère libre [13]. C'est ainsi qu'une grande partie du méthane produit en profondeur en conditions anoxiques est oxydé en $\mathrm{CO}_{2}$ lorsqu'il traverse les couches superficielles du sol ou d'eau (rizières) [14].

\subsection{Conclusion : nécessité d'une approche intégrée}

Pour analyser ou estimer les émissions de ces divers composés, on est donc toujours amené à considérer à la fois le climat, le sol et le milieu vivant (plantes ou micro-organismes). Il est nécessaire d'utiliser une approche physique (transferts de masse et d'énergie, équilibres physico-chimique) et biologique (fonctionnement des plantes et des micro-organismes). Il faudrait ajouter à cela un facteur humain, via les techniques agricoles, qui peuvent fortement limiter ou augmenter les risques d'émissions. Par exemple, l'enfouissement des engrais $[1,15]$ ou des pesticides [16] réduit considérablement le taux de volatilisation.

\section{DÉPÔT À LA SURFACE}

Le dépôt est un processus clef pour évaluer l'impact des activités agricoles sur l'environnement, puisqu'il représente la phase de retour des composés présents dans l'atmosphère vers les écosystèmes et les eaux superficielles. Le dépôt peut se faire par voie sèche (gaz, particules) ou humide (pluie, brouillard). Les quantités totales déposées peuvent être très importantes et représenter plusieurs dizaines de $\mathrm{kg}$ par hectare et par an (Tableau 1).

\begin{tabular}{|c|ccc|}
\hline Composé & $\begin{array}{c}\text { Dépôt total à } \\
\text { découvert }\end{array}$ & Dépôt humide & $\begin{array}{c}\text { Dépôt humide sous } \\
\text { couvert }\end{array}$ \\
\hline $\mathrm{H}^{+}$ & 0,5 & 0,7 & 1,1 \\
$\mathrm{~K}^{+}$ & 39,1 & 5,8 & 73,6 \\
$\mathrm{Ca}^{2+}$ & 47,0 & 15,7 & 48,2 \\
$\mathrm{Mg}^{2+}$ & 30,2 & 3,8 & 13,3 \\
$\mathrm{Na}^{+}$ & 137,0 & 45,6 & 61,8 \\
$\mathrm{~N}-\mathrm{NH}_{4}{ }^{+}$ & 43,6 & 12.2 & 32,6 \\
$\mathrm{~N}-\mathrm{NO}_{3}^{-}$ & 25,9 & 9,1 & 32,6 \\
$\mathrm{~S}-\mathrm{SO}_{4}{ }^{2-}$ & 47,5 & 19,6 & 94,6 \\
$\mathrm{Cl}^{-}$ & 436,0 & 58,9 & 64,1 \\
\hline
\end{tabular}

Tableau 1. - Dépôts maximaux de divers composés minéraux (en kg ha-lan-1). Mesures réalisées en France sur les réseaux BAPMON, MERA et DEMENT (d'après [6]).

Ces mesures ont été obtenues à partir d'analyses des eaux de pluies recueillies sur différents réseaux d'observation. Les auteurs observent une forte variabilité inter-annuelle liée principalement aux précipitations. Mais ces mesures ne prennent pas directement en compte les dépôts secs, même si une partie des dépôts mesurés sous couvert (colonne de droite) peut correspondre au lessivage des dépôts secs présents sur la cuticule des plantes. Les dépôts totaux réels sont donc supérieurs à ces valeurs. Pour l'azote ammoniacal par exemple, le dépôt sec représente à peu près la moitié des dépôts totaux à l'échelle de l'Europe [7].

\subsection{Dépôt humide}

Les composés déposés par voie humide sont des aérosols 
ou des gaz présents dans l'atmosphère qui se sont (1) dissous dans les gouttelettes des nuages ou des brouillards, ou (2) ont été lessivés de l'atmosphère au moment des précipitations. Le premier processus est le plus important quantitativement pour les composés gazeux, sauf à proximité des sources où la dissolution n'a pas eu le temps de se produire. La dissolution des gaz dans les gouttelettes dépend de leur solubilité, et de la taille, de la composition chimique et du $\mathrm{pH}$ des gouttes. Ainsi l'absorption des molécules d'ammoniac est-elle fortement favorisée par l'acidité générale des eaux de pluie. Le dépôt humide d'aérosols se fait principalement par collecte des aérosols (incorporation dans les gouttes suite à l'impact) lors des précipitations.

Le dépôt dû au brouillard est très variable selon les régions. C'est en grande partie le nombre de jours de brouillard, en général corrélé avec l'altitude, qui détermine le pourcentage du dépôt qu'on peut lui attribuer. On peut observer de forts dépôts dus au brouillard même s'il n'occasionne que des précipitations faibles [6]. La concentration des gouttelettes de brouillard peut en effet être beaucoup plus élevée que celle des pluies, en raison notamment de la petite taille des gouttelettes. La structure de la végétation est également importante car elle fait varier la surface de captation des gouttelettes.

\subsection{Dépôts secs}

On exprime en général le dépôt sec sous la forme du produit d'une vitesse de dépôt, par la concentration dans l'atmosphère :

$$
D=V_{d}\left[C_{a}-C_{o}\right]
$$

où $D$ est le dépôt $\left(\mu \mathrm{g} \mathrm{m}^{-2} \mathrm{~s}^{-1}\right), V_{d}$ la vitesse de dépôt $\left(\mathrm{m} \mathrm{s}^{-1}\right)$ et $C_{a}$ la concentration dans l'atmosphère $\left(\mu \mathrm{g} \mathrm{m}^{-3}\right)$. La concentration à la surface ou dans la végétation, ou point de compensation, $C_{o}$, n'est définie que pour le dépôt sec de certains composés gazeux ( $C_{o}=0$ pour les particules). Contrairement aux émissions, la concentration atmosphérique est ici déterminante car $C_{a}$ et $C_{o}$ sont souvent proches. Il n'y aura dépôt que si $C_{a}>C_{o}$, et le dépôt est d'autant plus fort que $C_{a}$ est élevée. Le point de compensation étant souvent faible, le dépôt peut être presque continu dans les zones polluées, ce qui peut conduire à des dépôt cumulés importants malgré des flux de dépôt journaliers généralement faibles (quelques dizaines ou centaines de $\mathrm{g} \mathrm{ha}^{-1}$ jour $^{-1}$ ).

\subsubsection{Particules}

Les vitesses de dépôt des particules dépendent principalement de leurs propriétés physiques, parmi lesquelles le diamètre est le plus important [8]. Les très petites particules (diamètre $<0,1 \mu \mathrm{m}$ ) se déposent principalement par diffusion brownienne et les grosses particules (diamètre $>2 \mu \mathrm{m}$ ) par gravitation. Les particules dont les diamètres sont compris entre 0,1 et $1 \mu \mathrm{m}$ se déposent sous l'effet de processus tels que l'interception ou l'impaction. En raison de leur forte vitesse de dépôt, les grosses particules (diamètre $>10 \mu \mathrm{m}$ ) vont rapidement disparaitre de l'atmosphère. Les gouttelettes de produits phytosanitaires épandus (quelques dizaines de $\mu \mathrm{m})$ ne vont ainsi se disperser qu'à quelques mètres ou dizaines de mètres de leur point d'émission, sauf si l'évaporation diminue suffisamment leur diamètre.

Pour le dépôt des petites particules, les caractéristiques de la surface sont déterminantes. Les facteurs-clefs sont la hauteur de la végétation, la longueur de rugosité, la surface foliaire, la taille des éléments collecteurs (feuilles, aiguilles), les propriétés d'adhésion de la surface et son état d'humidité. Mais la vitesse de dépôt est également fortement dépendante des conditions de turbulence. En air calme, la vitesse de chute d'une particule résulte de l'équilibre entre les forces de gravité et la traînée aérodynamique. Mais la convection étant presque toujours turbulente dans les basses couches atmosphériques, les temps de chute peuvent être très différents de ceux que laisserait prévoir une simple prise en compte de la vitesse de chute. De plus, dans un couvert végétal, la turbulence augmente fortement la probabilité que la particule soit captée, en augmentant sensiblement le trajet dans la végétation.

\subsubsection{Composés gazeux}

Le dépôt sec de composés gazeux dépend également des propriétés physiques et chimiques du gaz $[17,18]$. Pour des composés qui réagissent rapidement avec d'autres substances à la surface, le point de compensation, $C_{o}$, peut être considéré comme nul. Pour les gaz dissous dans l'eau interstitielle des plantes, il dépend de la température qui modifie sensiblement la constante de Henry, et il peut être aussi contrôlé par le pH dans la plante ou à sa surface. Pour expliciter son déterminisme, on exprime souvent la vitesse de dépôt de composés gazeux sous forme d'analogie électrique [18] (Fig.2) :

$$
V_{d}=l r_{a}+r_{b}+r_{c} J^{l}
$$

Ces trois résistances (en $\left.\mathrm{s} \mathrm{m}^{-1}\right)$ sont relatives à trois processus impliqués dans le dépôt. La résistance aérodynamique, $r_{a}$, dépend principalement de la turbulence locale, équilibre entre les conditions météorologiques et les caractéristiques aérodynamiques et thermiques de la surface. La résistance de la couche pseudo-laminaire au contact de la surface, $r_{b}$, dépend à la fois de la turbulence, de la structure du couvert végétal et de la diffusion moléculaire du composé considéré. La résistance de surface, $r_{c}$, dépend, elle, du composé et des caractéristiques de la surface réceptrice. Elle peut intégrer un assemblage complexe de (1) la résistance stomatique pour les gaz absorbés et métabolisés par la plante, et de (2) la résistance cuticulaire ou de la résistance de l'eau présente à la surface [18]. Ces dernières résistances peuvent intégrer des réactions chimiques avec d'autres composés précédemment déposés, au niveau de la cuticule ou de gouttelettes présentes à la surface de la végétation [17]. On sait notamment que les dépôts d'ammoniac et de dioxyde de soufre se favorisent l'un l'autre car chacun neutralise les variations de $\mathrm{pH}$ de la surface provoquées par l'autre [18].

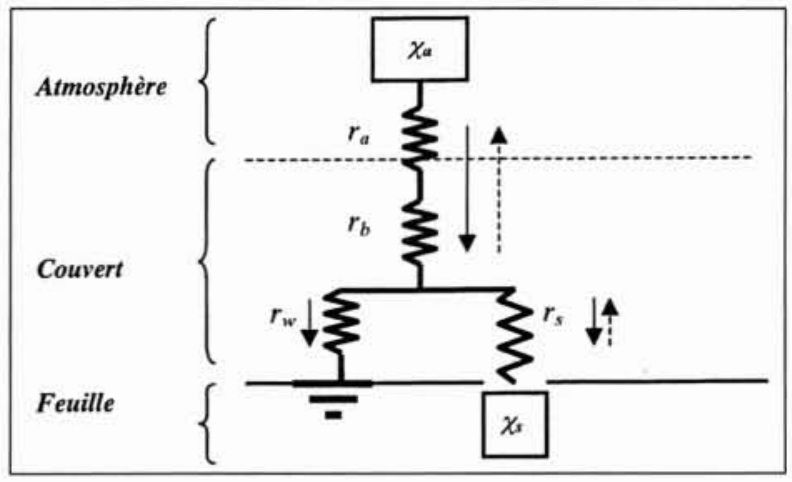

2. Résistances impliquées dans le calcul de la vitesse de dépôt. 
Les composés gazeux ou particulaires déposés à la surface de la végétation peuvent être ré-émis vers l'atmosphère, absorbés et métabolisés par la plante, transformés chimiquement ou lessivés vers le sol puis les eaux souterraines par les précipitations. Cela explique que les dépôts humides soient souvent plus importants sous couvert qu'en dehors du couvert (voir Tableau 1).

\section{TRANSFERT ATMOSPHÉRIQUE}

Qu'un composé soit sous forme particulaire (solide ou liquide) ou sous forme gazeuse, ce sont les mouvements convectifs de l'atmosphère qui sont les moteurs de son transport dans l'atmosphère. Même s'ils ne peuvent être dissociés, on peut distinguer deux processus liés à ces transferts convectifs. Tout d'abord, à proximité de la source, une dispersion très efficace qui fait diminuer rapidement la concentration des composés émis en fonction de la distance à la source et ensuite un transport rapide des composés sur des distances qui peuvent être grandes (plusieurs dizaines à centaines de km par jour).

Les échelles de temps de ces transferts sont beaucoup plus courtes que celles qui concernent les transferts dans le sol. Elles sont à la fois plus longues que les mouvements turbulents qui déterminent les échanges entre la biosphère et l'atmosphère (périodes de quelques centièmes de secondes à quelques minutes) et plus courtes que le cycle journalier. Pour le transport à longue distance, il faut toutefois considérer des échelles de temps plus grandes, limitées par la durée de vie du composé qui dépend de sa réactivité chimique et de son dépôt.

On a vu dans la partie précédente que la variable déterminante pour quantifier le dépôt était la concentration en gaz ou particules près de la surface du sol. Son évolution en fonction de la distance à la source est en général sous la dépendance des conditions météorologiques, des caractéristiques de la surface (rugosité) et, le cas échéant, de l'importance du dépôt entre la source et la " cible". Nous allons analyser ci-dessous l'influence de deux facteurs locaux agissant sur la dispersion et le dépôt de polluants.

\subsection{Rugosité de la surface}

La rugosité, que l'on peut relier aux caractéristiques géométriques de la surface du sol ou de la végétation (hauteur du couvert végétal ou des aspérités, densité foliaire,...) explique en partie l'intensité des échanges turbulents dans les basses couches atmosphériques. Plus la rugosité est forte et plus le mélange et la dispersion vont être efficaces. On peut estimer la hauteur de la couche limite qui se développe au-dessus d'une surface homogène $\left(h_{C L}\right)$ en fonction de sa rugosité $\left(z_{o}\right)$. A une distance de $x=100 \mathrm{~m}$, on aurait $h_{C L} \approx 12 \mathrm{~m}$ au dessus d'une prairie fauchée $\left(z_{o} \approx 0,01 \mathrm{~m}\right)$ et $h_{C L} \approx 34 \mathrm{~m}$ au-dessus d'une forêt $\left(z_{o} \approx 1 \mathrm{~m}\right)$. A grande distance d'une source, la rugosité favorise le dépôt en augmentant les échanges convectifs. Par contre, à proximité de la source, son effet sera le résultat de 2 actions contradictoires : une augmentation de la vitesse de dépôt par diminution de la résistance aérodynamique ( $r_{a}$ de la formule 3 ), mais une diminution de la concentration atmosphérique par accroissement de la dispersion.

\subsection{Stabilité thermique de l'atmosphère}

L'intensité de la turbulence et l'épaisseur de mélange sont aussi fortement dépendantes des conditions thermiques des basses couches atmosphériques. Les différences de température entre la surface et l'atmosphère induisent des différences de densité de l'air en fonction de l'altitude qui vont augmenter les échanges pendant le jour (conditions instables : la température est plus élevée à la surface) et les diminuer de nuit (conditions stables) par l'action de forces de poussée hydrostatique (Figure 3 ). De jour, les coefficients d'échange convectif peuvent ainsi être 2 à 3 fois plus forts qu'en conditions neutres. Toute perturbation ou émission à la surface se transmet rapidement dans l'atmosphère sur des épaisseurs importantes (un à plusieurs $\mathrm{km}$ ). Du fait de cette " dilution" rapide dans des volumes très importants, les concentrations décroissent rapidement. De nuit, à l'inverse, les gradients de température positifs diminuent les échanges. Lorsque le ciel est clair (fort déficit d'énergie par rayonnement), on peut ainsi aboutir à des situations où les échanges sont presque bloqués. Dans ce cas, les polluants sont dispersés sur des couches de faible épaisseur (quelques dizaines de mètres), et les concentrations vont rester fortes près de la surface du sol, même si les émissions sont faibles. Des phénomènes de drainage d'air froid le long des pentes peuvent également transporter et concentrer les polluants vers les zones basses, qui sont souvent des zones humides.

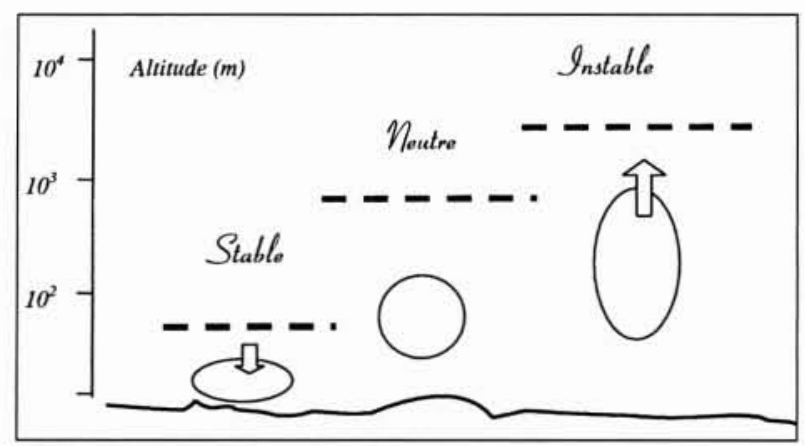

3. Conditions de stabilité et hauteur de la couche limite (les flèches symbolisent les forces de flottabilité).

\subsection{Modélisation de la dispersion atmosphérique}

Pour comprendre les déterminants d'un épisode de pollution ou interpréter des données de concentration mesurées en un point, il est nécessaire de faire appel à la modélisation des transferts atmosphériques. Les modèles existants sont nombreux et de niveaux de complexité très divers. Le choix d'un modèle dépend essentiellement des objectifs, des moyens expérimentaux mis en œuvre pour le valider, et des mesures disponibles pour le mettre en œuvre. On peut distinguer deux grands concepts de modélisation, qui font référence à 2 schémas de référence mathématique : l'approche eulérienne dans laquelle les mouvements atmosphériques sont référencés dans un système fixe par rapport à la surface terrestre, et l'approche lagrangienne où le système de référence suit le mouvement moyen de l'atmosphère.

Dans un modèle eulérien, la dynamique de l'écoulement atmosphérique est donnée par les équations de Navier-Stokes, complétées par les équations de continuité et de conservation de l'énergie. Quelques simplifications aboutissent au système de Boussinesq. De tels modèles permettent de calculer les différentes variables impliquées dans la dispersion et le transport de polluants dans l'atmosphère (champs de vent dans les trois dimensions $(u, v, w)$, température et concentrations) et 
leurs évolutions suivant des échelles de temps et d'espace variables selon le problème posé : de quelques dizaines de mètres pour des problèmes de dispersion locale d'un polluant, à l'échelle continentale pour des problèmes de transports transfrontaliers de pollution. Un des intérêts des modèles eulériens est que les variables eulériennes sont mesurables et que les formulations sont applicables dans les situations où se produisent des réactions chimiques. Le principal problème concerne la fermeture des équations : on peut utiliser des modèles diffusifs simples (fermeture d'ordre 1) ou des modèles à fermeture d'ordre 2 ou 3 .

Dans un modèle lagrangien, la concentration en un lieu et temps donnés est la somme des contributions de toutes les sources avoisinantes, chacune étant pondérée par une fonction de densité de probabilité qu'un volume d'air se déplace d'un point à un autre dans l'espace. Cette approche peut s'appliquer d'une échelle très locale à l'échelle globale. Pour les problèmes de pollution, elle permet de bien prendre en compte la dispersion à proximité des sources. La difficulté réside dans la détermination des fonctions de densité de probabilité. Les différents modèles ont pour cela des niveaux de complexité très différents, allant du modèle de panache gaussien aux modèles lagrangiens stochastiques.

Pour estimer les dépôts à l'échelle d'une région ou d'un pays, ces modèles sont couplés à des modèles ou à des cadastres d'émission et à des modèles de dépôt $[7,8]$.

\section{CONCLUSIONS}

Le transfert atmosphérique prend de multiples formes et peut être un facteur déterminant de contamination des eaux. Un exemple en est la détérioration de nombreux lacs scandinaves sous l'influence des dépôts atmosphériques de composés soufrés notamment. Pour comprendre tous les déterminants du problème, il est nécessaire de prendre en compte à la fois les processus d'émission, de transport et de dépôt en mettant particulièrement l'accent sur les interactions entre l'atmosphère et la surface. En effet pour comprendre l'impact des activités agricoles sur un écosystème donné, ou un plan d'eau, il faut connaître la répartition des sources, leur intensité et les relations entre les sources et la cible, c'est-à-dire la probabilité qu'une molécule d'un composé émis sur une source arrive au contact d'un écosystème ou des eaux superficielles. Cette probabilité, ainsi que les échelles d'espace et de temps à considérer, dépend des conditions de dispersion et de transport atmosphérique, ainsi que du composé lui-même (densité d'émission, réactivité, vitesse de dépôt) et des caractéristiques de l'environnement.

\section{Bibliographie}

[1] Génermont S., 1996. Modélisation de la volatilisation d'ammoniac après épandage de lisier sur parcelle agricole. Thèse de Doctorat, Univ. Toulouse, INRA Bioclimatologie Grignon, 331p.

[2] Pimentel D., Levitan L., 1986. Pesticides : amount applied and amounts reaching pests. Bioscience, $36: 86-91$.

[3] Van den Berg F., 1992. Emission of fumigants from soil and dispersion in air. Thèse, Univ. Wageningen, Pays-Bas, 224 p.

[4] Jansma J.W., Linders J.B.H.J., 1995. Volatilization of pesticides from soil and plants after spraying. Report No 679102030, R.I.V.M., Bilthoven, Pays-Bas, $48 \mathrm{p}$

[5] Paterson S., MacKay D., Tham D., Shiu W.Y., 1990. Uptake of organic chemicals by plants : a review of processes, correlations and model. Chemosphere, $21: 297-331$

[6] Ulrich E. et Williot B., 1993. Les dépôts atmosphériques en France de 1850 à 1990. INRA Ed., Paris, 130 p. + Annexes.

[7] Asman W.A.H., van Jaarsveld, J.A., 1992. A variable-resolution transport model applied for NHx in Europe. Atmos. Environ. 26A : 445-464.

[8] Van Jaarsveld J.A., 1995. Modelling the long-term atmospheric behaviour of pollutants on various spatial scale. Thèse, Univ. Utrecht, PaysBas, $235 \mathrm{p}$.

[9] Fontelle J.-P., Chang J.P., Allemand N., Audoux N., Levy C., Rivière E., 1998. Inventaire des émissions dans l'air en France au cours de la période 1990-96. CITEPA

[10] Adriaanse P.I., 1996. Fate of pesticides in field ditches : the TOXSWA simulation model. Report 90, SC-DLO, Wageningen, Pays-Bas, 124 p. + Annexes.

[11] Marquis A., Marchal P., 1998. Qualité de l'atmosphère à proximité des bâtiments d'élevage. Cahiers Agriculture, 7, 377-385.

[12] Génermont S., Cellier P., 1997. A mechanistic model for estimating ammonia volatilization from slurry applied to bare soil. Agric. For. Meteorol., 88, 145-167.

[13] Renault P., Parry S., Sierra J., Bidel L., 1997. Transferts de gaz dans les sols. Courrier de l'Environnement, 32 : 33-50.

[14] Conrad R., 1989. Control of methane production in terrestrial ecosystems. In : M.O. Andreae et D.S. Schimel (Eds.) Exchange of trace gases between terrestrial ecosystems and the atmosphere, J. Wileys and Sons Ltd, Chichester, 39-58.

[15] Jarvis S.C. et Pain B.F., 1990. Ammonia volatilization from agricultural land. Fert. Soc. Proc. no. 298. 25p.

[16] Van der Werf H., 1997. L'impact des pesticides sur l'environnement. Courrier de l'Environnement, $31: 5-22$.

[17] Fléchard, C.R., Fowler, D., Sutton, M.A. and Cape, J.N. (1999). A dynamic chemical model of bi-directional ammonia exchange between semi-natural vegetation and the atmosphere. Quart. J. Roy. Meteorol. Soc. (sous presse)

[18] Sutton M.A., Schjoerring J.K ., Wyers G.P., 1995. Plant-atmosphere exchange of ammonia. Phil. Trans. Roy : So. Soc., London, Series A. $351: 261-278$. 